\title{
BMJ Open Mindful Self-Care and Resiliency (MSCR): protocol for a pilot trial of a brief mindfulness intervention to promote occupational resilience in rural general practitioners
}

Clare Rees, ${ }^{1}$ Mark Craigie, ${ }^{1}$ Susan Slatyer, ${ }^{2,3}$ Brody Heritage, ${ }^{4}$ Clare Harvey, ${ }^{5}$ Paula Brough, ${ }^{6}$ Desley Hegney ${ }^{5}$

To cite: Rees C, Craigie M, Slatyer S, et al. Mindful SelfCare and Resiliency (MSCR): protocol for a pilot trial of a brief mindfulness intervention to promote occupational resilience in rural general practitioners. BMJ Open 2018;8:e021027. doi:10.1136/ bmjopen-2017-021027

\section{- Prepublication history for} this paper is available online. To view these files please visit the journal online (http://dx.doi. org/10.1136/bmjopen-2017021027).

Received 12 December 2017 Revised 26 April 2018 Accepted 21 May 2018

\section{Check for updates}

${ }^{1}$ School of Psychology and Speech Pathology, Curtin University, Perth, Western Australia, Australia

${ }^{2}$ School of Nursing, Midwifery and Paramedicine, Curtin University, Perth, Australia ${ }^{3}$ Centre for Nursing Research, Sir Charles Gairdner Hospital, Perth, Western Australia, Australia

${ }^{4}$ School of Psychology, Murdoch University, Perth, Australia

${ }^{5}$ Research Division, Central Queensland University, Brisbane, Australia

${ }^{6}$ School of Applied Psychology, Griffith University, Nathan, Queensland, Australia

Correspondence to

Professor Clare Rees;

C.Rees@curtin.edu.au

\section{ABSTRACT}

Introduction The Mindful Self-Care and Resiliency (MSCR) programme is a brief psychosocial intervention designed to promote resilience among various occupational groups. The intervention is based on the principles of mindfulness and also incorporates an educational self-care component. The current paper presents the protocol for a pilot study that will evaluate the effectiveness of this programme among general practitioners working in rural Queensland, Australia.

Methods and analysis We will measure the impact of the MSCR programme on levels of employee resilience (Connor-Davidson Resilience Scale; State-Trait Assessment of Resilience STARS), compassion satisfaction and compassion fatigue (Professional Quality of Life Scale), self-compassion (Self-Compassion Scale) and mood (Positive and Negative Affect Scale). We will also assess the impact of the programme on job satisfaction (The Abridged Job in General Scale), absenteeism/presenteeism (The WHO Health and Work Performance Questionnaire) and general well-being (WHO Five Well-being Index). Repeated measures analysis of variance will be used to analyse the impact of the intervention on the outcome measures taken at pre, post, 1-month, 3-month and 6-month follow-ups. We will conduct individual interviews with participants to gather data on the feasibility and acceptability of the programme. Finally, we will conduct an initial cost-effectiveness analysis of the programme.

Ethics and dissemination Approval for this study was obtained from the Curtin University Human Research ethics committee and the study has been registered with the Australian Clinical Trials Registry. Results will be published and presented at national and international congresses. Trial registration number ACTRN12617001479392p; Pre-results.

\section{BACKGROUND}

The work of a general practitioner (GP) is highly demanding, requiring the provision of high-quality continuity of care to a wide spectrum of patients while often under significant time pressure. It is of concern

\section{Strengths and limitations of this study}

- This study is the first trial of this intervention with a sample of general practitioners.

- In addition to assessing change on standardised measures, this study will assess acceptability and feasibility by including individual interviews with participants.

- The study uses several follow-up evaluations (1, 3 and 6 months postintervention).

- As this is an initial pilot study, there is no control group comparison limiting firm conclusions that changes are attributable to the intervention itself.

- The nature of the sample (rural, Australian) means that results may have limited generalisability to broader groups of general practitioners.

that approximately $50 \%$ of physicians report experiencing burnout at some stage in their careers, ${ }^{1}$ and it is estimated that at any point in time, one in three doctors are suffering burnout. ${ }^{2}$ Unfortunately, the experience of burnout is strongly associated with increased medical errors and is highly correlated with decreased patient satisfaction, decreased employee job satisfaction, lower job productivity, increased absenteeism and intent to leave. $^{3}$

In addition to the already stressful nature of general practice, rural doctors face a further set of unique challenges. These include but are not limited to:

- Less access to professional development opportunities.

- Professional isolation; less collegial support available.

- Additional requirements of delivering care in remote locations, for example, having to commute long distances and work longer hours. 
It is therefore essential that GPs are provided with the skills they need to recognise when they are at risk of burnout and the kinds of strategies they can put in place to maintain their own self-care and emotional well-being. Our research group has developed an evidence-supported intervention that has been shown to reliably reduce burnout and improve well-being among nurses. This programme, the Mindful Self-Care and Resiliency (MSCR) intervention is a brief, skills-based programme that incorporates principles of mindfulness, self-care and self-compassion. We have published two trials of this programme with nurse participants, one an initial pilot investigation ${ }^{4}$ and a larger randomised controlled trial $^{5}(\mathrm{n}=91)$. Our programme is one of only nine mindfulness-based programmes for healthcare professionals that have been formally evaluated worldwide. ${ }^{6}$ Of these, only three others have been evaluated experimentally and, in contrast to our controlled trial, have included much smaller samples $(n=36, n=38, n=43)$ and limited follow-up periods. ${ }^{6}$ The brief nature of the MSCR programme (6.5 hours followed by 1.5-hour follow-ups weekly over the next 3 weeks) means that it is affordable to deliver and does not require a large time commitment, ensuring its feasibility for busy doctors and reductions in organisational backfill costs.

The present study, working in partnership with Central Highlands Healthcare and the Rural Doctors Association of Australia and the Generalist Medical Training Programme, provides a unique opportunity to directly build occupational well-being among rural doctors.

\section{Current intervention: development protocol}

Several preliminary steps were undertaken in the development of the MSCR intervention. First, we based the content of the intervention on existing explanatory models of adaptive emotion regulation and resiliency. Gentry and Baranowsky ${ }^{7}$ suggested that interventions that target adaptive emotion regulation and patterns of thinking in response to stressors and foster new ways of relating to work may be central to building resiliency and reducing compassion fatigue (CF). Furthermore, Rees and colleagues 8 proposed a model of workplace resilience that highlights the importance of mindfulness as an adaptive process that enables more balanced appraisals of stressful events. Mindfulness is defined as the awareness that emerges through paying attention on purpose, in the present moment and non-judgementally. ${ }^{9}$ The two most dominant mindfulness approaches are mindfulness-based stress reduction (Kabat-Zinn ${ }^{9}$ ) and mindfulness-based cognitive therapy (Segal et $a l^{10}$ ). Evidence from occupational settings shows that these 8-week interventions reliably lower stress ${ }^{11}$ and burnout ${ }^{1213}$ and increase job satisfaction. ${ }^{14}$ However, in busy occupational settings, the 8-week duration of conventional mindfulness-based interventions may limit their broader adoption. ${ }^{15}$ Fortunately, a small number of studies have shown that more brief and less intensive mindfulness-based interventions lead to significant reductions in burnout symptoms and increased resilience in nurses and healthcare workers. ${ }^{16-18}$

To address the above limitations, we developed an MSCR programme to be a brief psychosocial intervention that integrates key principles and practices of mindfulness-based therapy with CF prevention education as developed by Gentry and colleagues. ${ }^{719}$ We conducted a pilot study to test the feasibility of MSCR for nurses working at an acute care hospital. ${ }^{4}$ As part of this initial evaluation, we also interviewed the nurse participants to obtain their feedback as to the acceptability and effectiveness of the intervention. ${ }^{20}$ Finally, we compared the intervention to a wait-list control in a non-randomised wait-list control study. ${ }^{5}$ Both the initial pilot and the non-randomised control study showed that the MSCR intervention resulted in significant reductions in burnout and improvements on various indices of psychological well-being. The nurses interviewed reported that the intervention was relevant and the strategies taught were easy to apply to their workplace setting (eg, 3 min breathing space). The next step in development of the MSCR programme is to examine its feasibility, acceptableness and effectiveness with a sample of medical practitioners.

\section{Current intervention: research protocol}

Following the initial pilot studies, we developed a Standard Protocol Items: Recommendations for Interventional Trials (SPIRIT)compliant research protocol (see table 1) to evaluate the efficacy of the programme among a cohort of rural GPs.

\section{Aims and hypotheses}

The aim of this research trial is to investigate whether the MSCR intervention is effective in reducing burnout, secondary traumatic stress and symptoms of general psychological distress in GPs working in a rural setting in Queensland, Australia. Secondary aims are to determine whether the intervention increases protective factors for occupational stress, including compassion satisfaction, self-compassion and resilience and to evaluate the cost-effectiveness of the programme.

Our primary hypothesis is that doctors completing the MSCR intervention will show significant reductions in symptoms of burnout, secondary traumatic stress and general psychological distress from preintervention to postintervention and from preintervention to follow-up. A secondary hypothesis is that doctors completing the MSCR intervention would show significant improvements in compassion satisfaction, self-compassion and resilience from preintervention to postintervention and from preintervention to follow-up.

\section{METHODS/DESIGN}

\section{Study design and data analysis}

We will conduct a pilot investigation of the effectiveness of the MSCR programme with GPs using a sequential mixed-method study design. Mixed-method designs use 
Table 1 Items from the WHO trial registration

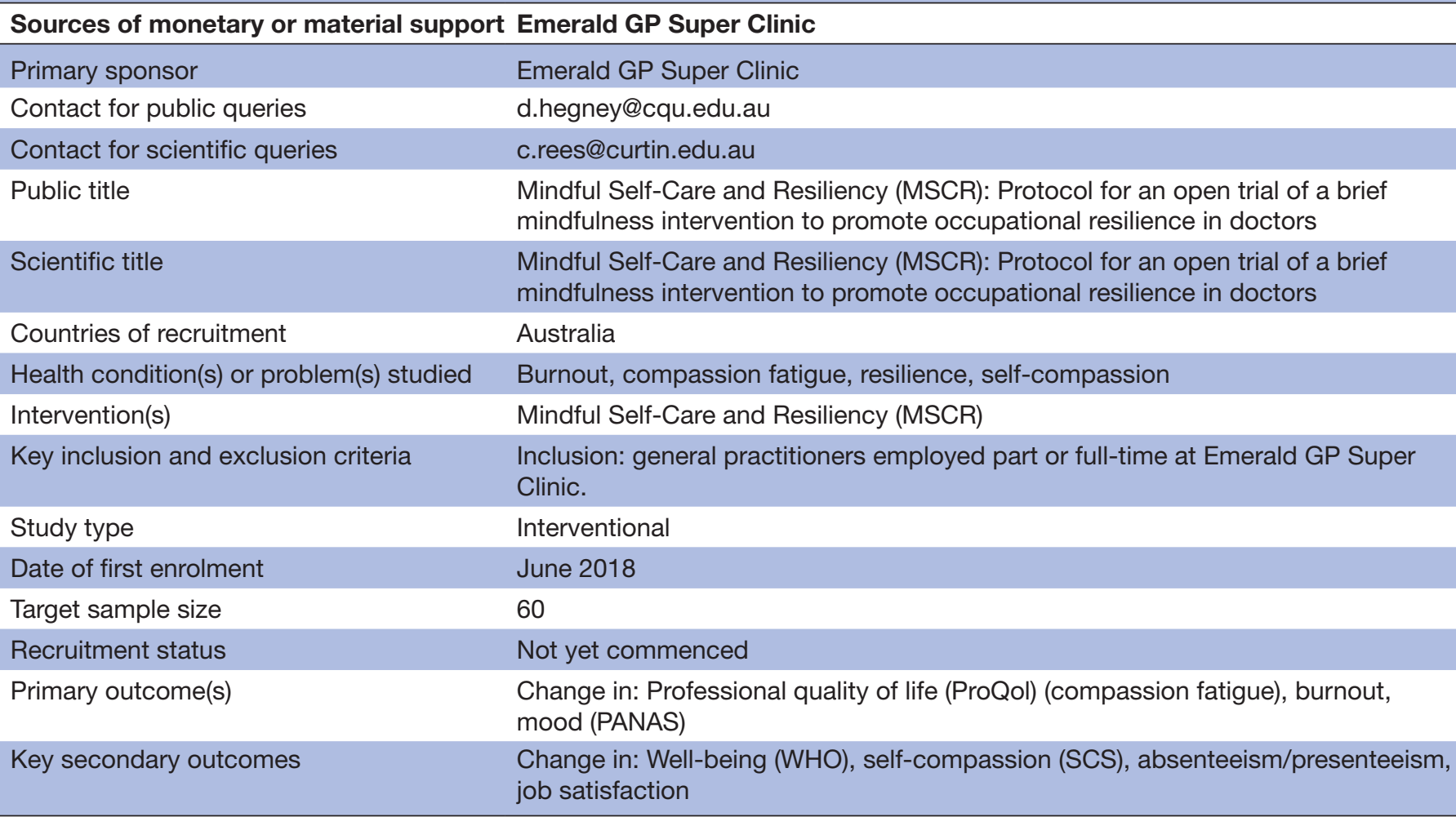

PANAS, Positive and Negative Affect Scale; ProQol, Professional Quality of Life Scale; SCS, Self - Compassion Scale.

a blend of qualitative and quantitative data collection, procedures and analysis. This design provides data for a deeper understanding of occupational resilience and the acceptability, feasibility, applicability and effectiveness of the MSCR programme to build and maintain resilience among rural GPs.

Effectiveness of the programme: will be assessed by examining changes in scores on outcome measures at pre, post, 1-month, 3-month and 6-month follow-ups.

Feasibility, acceptability and applicability of the MSCR programme: will be determined using open-ended individual telephone interviews (approximately $30 \mathrm{~min}$ ) with a purposive sample of 20 participants, 1 month following the intervention. While it is envisaged that 20 participants will provide data saturation, if this is not reached, interviews will continue until it is reached, or the pool is exhausted. Interviews will be transcribed verbatim and subjected to thematic analysis. An experienced qualitative researcher (Hegney) will conduct the interviews, and then independently read the transcripts line by line to identify meaningful words and phrases, which will be coded to generate categories in the data. These categories will be grouped and collapsed to develop overarching themes describing responses to the MSCR programme. Further coding to identify subthemes will be conducted using NVivo qualitative analysis software. Emergent themes and subthemes will be presented in narrative form.

Cost-effectiveness of the programme: The cost analysis will be used to estimate the associated costs of the programme, while outcomes will be expressed as cost per sick leave avoided and cost per quality-adjusted life years gained. The Assessment of Quality of Life (AQol) 8D will be used to collect quality of life data. The AQol-8D is a health-related quality of life instrument that has been designed for use in economic evaluation studies. ${ }^{21}$ The advantage of this instrument, above others, is that it was designed to capture changes in both physical and psychosocial health and it is simple and quick to administer. The AQoL-8D will be administered at 1 month and 6 months follow-up. Quality of life values will be collated using the appropriate algorithms as outlined on the AQol website.

\section{Sample size}

We aim to recruit 60 participants. A priori sample size estimation assuming at least an $80 \%$ chance of detecting a moderate anticipated effect size $(f=0.25)$ at an alpha level of 0.05 suggests that a total sample of $n=32$ will be sufficient. Our previous studies ${ }^{45}$ suggest a $10 \%$ attrition rate across the study. Our initial intake of 60 participants will be sufficient to achieve the minimum sample size requirement of $n=32$ by the final follow-up.

\section{Participants}

Inclusion criteria: GPs and registrars working in either a full-time or part-time capacity in Emerald, Queensland. Emerald has a population of 13500 and has one rural hospital and seven medical centres. The super clinic is the largest of the medical practices and is an accredited practice. 


\section{Recruitment}

GPs and registrars from the Emerald, Queensland will be recruited to take part. The Emerald Super clinic approached the researchers to conduct the study. Convenience sampling will be used to recruit GPs to participate in one of two MSCR programmes (30 participants in each).

\section{Procedure}

All GPs and registrars employed in one of the medical practices in Emerald will be invited to take part in the programme. An email will be sent from the Emerald Super clinic to all of the Emerald practices outlining the start date of the programme and providing a summary of the research aims. The email will outline the nature of the intervention (resilience building and mindfulness training) and the time commitment required if they decide to participate. This email will make it clear that they can attend the programme and opt not to take part in the research component if they wish. Data collection points are: immediately before and after the face-to-face session (hard copy survey); on completion of third follow-up session (1 month), 3 months and 6 months. On arrival at the MSCR groups, participants will be welcomed and the study explained verbally and envelopes handed out with Participant Information Sheet and consent forms as well as the outcome measures to be completed. Participants will simply place their completed forms into a secure box at the back of the room. Those not taking part in the research will simply post their uncompleted forms. The 1-day face-to-face MSCR session will be conducted at a Rural Medical Resilience Retreat in April 2018; the three 1.5-hour follow-up sessions will be conducted using a video conference software as it will provide a secure environment and overcomes the need for participants working in rural and remote sites to travel long distances to meet face to face.

\section{Intervention: MSCR protocol}

The MSCR programme will be facilitated by Dr Craigie (the developer of the MSCR programme), who has worked closely with our team on previous studies. The full-day workshop includes education about CF resiliency and introduces participants to mindfulness concepts (eg, Autopilot Metaphor; Staying Present) and basic practices (eg, breath meditation). Each participant will be provided with a manual that includes materials about CF and its causes, and skills to build CF resiliency, referred to as the five 'antibodies' (1-self-regulation; 2-intentionality; 3-perceptual maturation; 4-connection and social support; and 5-self-care and revitalisation). Both formal (eg, body and breath; body scan) and informal practices (eg, breathing space) are included and assigned as home-based practice exercises. A full description of the MSCR session content is provided in Craigie. ${ }^{5}$ Participants will repeat the outcome measures again at 1-month, 3-month and 6-month follow-ups (electronically via Qualtrics).

\section{Outcome measures}

Demographics: (eg, age, gender, qualifications, number of years working for employer)

The Connor-Davidson Resilience Scale ${ }^{22}$ is a valid and reliable measure of resilience for normal and clinical populations.

The Burnout Measure-Short Version (BMS) is a short 10 -item version of the widely used 21-item scale. Data from two national samples (Israeli Jewish and Arab) and three occupational samples attest to the validity and reliability of the BMS. ${ }^{23}$

Positive and Negative Affect Scale (PANAS) ${ }^{24}$ is a 20-item measure of affective state that uses a 5-point Likert scale. It has two subscales: one measuring positive affectivity and the other negative affectivity. The PANAS has demonstrated excellent reliability and validity, and is frequently used in research and clinical settings.

Cognitive and Affective Mindfulness Scale, Revised ${ }^{25}$ is a 20 item self-report measure of trait-based mindfulness.

The Self-Compassion Scale-Short Form $(\mathrm{SCS}-\mathrm{SF})^{26}$ is a brief 12-item scale measuring self-kindness, common humanity and mindfulness, defined as holding one's experience in a balanced perspective. The SCS-SF consists of six subscales measuring self-kindness, self-judgement, common humanity, feelings of isolation, mindfulness and overidentification (the tendency to fixate on negative perceptions of self). Each item is structured as a statement describing a particular behaviour. The respondent is asked to indicate how often he or she behaves in this stated manner on a five-point scale from 'Almost never' to 'Almost always'. Negative constructs (self-judgement, isolation and overidentification) are reversed scored and the total mean calculated to obtain a total self-compassion score. Higher scores indicate more self-compassion in difficult times.

Cybernetic Coping Scale is a 14-item measure of $\operatorname{coping}^{27}$ with a 6-point response format that acknowledges the function of coping as both an independent variable and an outcome.

The Professional Quality of Life Scale version 5 (ProQol5) $^{28}$ provides a measure of the constructs of compassion satisfaction and CF; the second of which comprises burnout and secondary traumatic stress. Compassion satisfaction indicates an individual's positive adjustment to work derived from the pleasure of caring for others and the sense of performing well. On the other hand, burnout manifests as feelings of frustration, depressed mood and exhaustion that arise as negative aspects of caring. The third construct, secondary traumatic stress, constitutes avoidance behaviours in response to work-related trauma associated with exposure to others' suffering through caring. The ProQol5 is a 30-item, fivepoint Likert scale, containing three 10-item subscales measuring each of the aforementioned constructs.

The WHO (Five) Well-being Index (WHO Five) ${ }^{29}$ is a short instrument measuring subjective quality of life based on positive mood, vitality and general interest. It has five items rated on a six-point Likert scale. Percentage 
scores are reported and compared with monitor changes in well-being. A difference of $10 \%$ indicates a significant change. The instrument has been well-validated in the general population. ${ }^{29}$

The WHO Health and Work Performance Questionnaire (WHO-HPQ) ${ }^{30}$ Absenteeism will be measured by four items from the WHO-HPQ. Kessler et al demonstrated significant relationships between the self-reported scale responses and objective records of missed work from payroll records. Job performance will be measured by two items from the WHO-HPQ's presenteeism subscale which asks respondents to rate their own performance, and the average performance of other workers in their field, to form a relative indication of job performance.

The Abridged Job in General Scale ${ }^{31}$ will be used to measure job satisfaction. This eight-item scale is a short version of the previous Job in General Scale. Participants respond with 'Yes', 'No', or '?' to affective descriptors of their job at the global level. Excellent measurement model validity and reliability indices have been demonstrated.

\section{Data analyses}

This study will use repeated measures analysis of variance to test the effectiveness hypotheses. Effect sizes will be calculated to derive a Cohen's $d$ coefficient. Thematic analysis will be used to analyse the qualitative interview data.

\section{Data collection and monitoring}

Data will be collected and managed in line with the Australian Code for the Responsible Conduct of Research.

\section{Ethics}

This protocol has received ethical approval.

\section{Methodological considerations}

As the MSCR intervention has not yet been trialled with GPs, a preliminary pilot trial is appropriate. We will also be conducting interviews with approximately 20 of the participants to acquire information on the feasibility and acceptability of the programme. Further, we will conduct a basic cost analysis to obtain some preliminary information on cost-effectiveness of the programme. The inclusion of three follow-up data collection points is also a strength because it will provide important information about the longer term impact of the intervention. We acknowledge that participants will be required to complete a substantial number of instruments and that this may impact on participation rates. However, our previous trials with nurses ${ }^{45}$ included a similar number of instruments and we were able to achieve excellent rates of participation and retention in the programme and at follow-up.

\section{Patient and public involvement}

The target participants for this project are GPs. The project design and aims were informed by discussions with key stakeholders (GP clinic executive) who requested the intervention in the context of professional development of rural doctors as part of their regular professional development training. An executive manager of the rural GP super clinic representing participants was involved in establishing the study context, aims, timing, recruitment and resourcing. Intervention participants were not involved in the recruitment to the study. Research outcomes will be disseminated in summary form via routine rural clinic communiques and staff seminars.

\section{DISCUSSION}

This paper presents the protocol of a pilot trial designed to assess the effectiveness of a brief intervention to reduce burnout and improve the resilience of GPs working in rural Australia. The results of this study are therefore likely to be most applicable to similar groups of GPs working in rural areas but may have limited generalisability to other groups of GPs (such as those working in urban settings). As this is a pilot trial of the intervention, the next step will be to conduct a larger study and include a control group comparison and a 12-month follow-up period. We also intend to trial the intervention with GPs working in urban settings.

It has been shown that rates of burnout among physicians are higher in those working in community settings compared with hospital-based settings. ${ }^{32}$ Therefore, the current trial aimed at reducing burnout in GPs working in a community setting is well justified. Effective interventions that can be implemented into busy workplaces are important if we are to support doctors to remain resilient in the face of workplace stress.

Contributors CSR and DH drafted the manuscript. MC and SS contributed to the study design. $\mathrm{BH}, \mathrm{PB}$ and $\mathrm{CH}$ contributed to the study methodology. All authors read and approved the final manuscript.

Funding The study is funded by the Emerald Super Clinic in Queensland, Australia. Competing interests None declared.

Patient consent Not required.

Ethics approval Curtin University Human Research Ethics Committee (HRE2017-0847).

Provenance and peer review Not commissioned; externally peer reviewed.

Open access This is an open access article distributed in accordance with the Creative Commons Attribution Non Commercial (CC BY-NC 4.0) license, which permits others to distribute, remix, adapt, build upon this work non-commercially, and license their derivative works on different terms, provided the original work is properly cited and the use is non-commercial. See: http://creativecommons.org/ licenses/by-nc/4.0/

(c) Article author(s) (or their employer(s) unless otherwise stated in the text of the article) 2018. All rights reserved. No commercial use is permitted unless otherwise expressly granted.

\section{REFERENCES}

1. Lee ME, Brown DW, Cabrera AG. Physician burnout: an emergent crisis. Prog Pediatr Cardiol 2017;44:77-80.

2. Paterson R, Adams J. Professional burnout - a regulatory perspective. N Z Med J 2011;124:40-60.

3. Dyrbye $\mathrm{L}$, et al. A call to explore and address this underrecognized threat to safe, high quality patient care: NAM (National Academy of Medicine) Perspectives, 2017.

4. Craigie M, Slatyer S, Hegney D, et al. A pilot evaluation of a mindful self-care and resiliency (mscr) intervention for nurses. Mindfulness 2016;7:764-74. 
5. Slatyer S, Craigie M, Heritage B, et al. Evaluating the Effectiveness of a Brief Mindful Self-Care and Resiliency (MSCR) intervention for nurses: a controlled trial. Mindfulness 2018;9:534-46.

6. Burton A, Burgess C, Dean S, et al. How effective are mindfulnessbased interventions for reducing stress among healthcare professionals? a systematic review and meta-analysis. Stress Health 2017;33:3-13.

7. Gentry JE, Baranowsky AB. Treatment manual for the accelerated recovery program. Toronto, ON: Psyche Ink Resources, 1998.

8. Rees CS, Breen LJ, Cusack L, et al. Understanding individual resilience in the workplace: the international collaboration of workforce resilience model. Front Psychol 2015;6.

9. Kabat-Zinn J. Mindfulness-based interventions in context: past, present, and future. Clinical Psychology: Science and Practice 2003;10:144-56.

10. Segal Z. V, Williams JMG, Teasdale JD. Mindfulness-based cognitive therapy for depression. New York, NY: The Guilford Press, 2002.

11. Wolever RQ, Bobinet KJ, McCabe K, et al. Effective and viable mindbody stress reduction in the workplace: a randomized controlled trial. J Occup Health Psychol 2012;17:246-58.

12. Goodman MJ, Schorling JB. A mindfulness course decreases burnout and improves well-being among healthcare providers. Int $\mathrm{J}$ Psychiatry Med 2012:43:119-28.

13. Ruths FA, de Zoysa N, Frearson SJ, et al. Mindfulness-based cognitive therapy for mental health professionals - a pilot study. Mindfulness 2013;4:289-95.

14. Hülsheger UR, Alberts HJ, Feinholdt A, et al. Benefits of mindfulness at work: the role of mindfulness in emotion regulation, emotional exhaustion, and job satisfaction. J Appl Psychol 2013;98:310-25.

15. Zeller JM, Levin PF. Mindfulness interventions to reduce stress among nursing personnel. Workplace Health Saf 2013;61:85-9.

16. Gauthier T, Meyer RM, Grefe D, et al. An on-the-job mindfulnessbased intervention for pediatric ICU nurses: a pilot. $J$ Pediatr Nurs 2015;30:402-9.

17. Mackenzie CS, Poulin PA, Seidman-Carlson R. A brief mindfulnessbased stress reduction intervention for nurses and nurse aides. Appl Nurs Res 2006;19:105-9.

18. Sood A, Prasad K, Schroeder D, et al. Stress management and resilience training among Department of Medicine faculty: a pilot randomized clinical trial. J Gen Intern Med 2011;26:858-61.
19. Flarity K, Gentry JE, Mesnikoff N. The effectiveness of an educational program on preventing and treating compassion fatigue in emergency nurses. Adv Emerg Nurs J 2013;35:247-58.

20. Slatyer S, Craigie M, Rees C, et al. Nurse experience of participation in a mindfulness-based self-care and resiliency intervention. Mindfulness 2018;9:610-7.

21. Richardson J, et al. Increasing the sensitivity of the AQoL inventory for the evaluation of interventions affecting mental health. Melbourne: Centre for Health Economics, Monash University, 2011.

22. Connor KM, Davidson JR. Development of a new resilience scale: the Connor-Davidson Resilience Scale (CD-RISC). Depress Anxiety 2003;18:76-82

23. Malach-Pines $A$. The burnout measure, short version. Int $J$ Stress Manag 2005;12:78-88.

24. Watson D, Clark LA, Tellegen A. Development and validation of brief measures of positive and negative affect: the PANAS scales. J Pers Soc Psychol 1988;54:1063-70.

25. Feldman G, Hayes A, Kumar S, et al. Mindfulness and emotion regulation: the development and initial validation of the Cognitive and Affective Mindfulness Scale-Revised (CAMS-R). J Psychopathol Behav Assess 2007;29:177-90.

26. Neff KD. The development and validation of a scale to measure selfcompassion. Self and Identity 2003;2:223-50.

27. Brough $P, O^{\prime}$ Driscoll $M$, Kalliath $T$. Evaluating the criterion validity of the Cybernetic Coping Scale: Cross-lagged predictions of psychological strain, job and family satisfaction. Work Stress 2005;19:276-92.

28. Stamm B. The Concise ProQOL Manual, 2010.

29. Bech $P$, Olsen LR, Kjoller M, et al. Measuring well-being rather than the absence of distress symptoms: a comparison of the SF-36 Mental Health subscale and the WHO-Five Well-Being Scale. Int J Methods Psychiatr Res 2003;12:85-91.

30. Kessler RC, Barber C, Beck A, et al. The World Health Organization Health and Work Performance Questionnaire (HPQ). J Occup Environ Med 2003;45:156-74.

31. Russell SS, Spitzmüller C, Lin LF, et al. Shorter can also be better: the abridged job in general scale. Educ Psychol Meas 2004;64:878-93.

32. Prosser D, Johnson S, Kuipers E, et al. Mental health, "burnout' and job satisfaction among hospital and community-based mental health staff. Br J Psychiatry 1996;169:334-7. 